\section{Functional upper airways obstruction: two patients with persistent symptoms}

\author{
Christopher J Warburton, Robert McL \\ Niven, Bernard G Higgins, C Anthony C \\ Pickering
}

\begin{abstract}
Functional upper airways obstruction is caused by vocal cord dysfunction and classically occurs in paroxysms closely resembling acute asthmatic attacks. We present two cases in which the symptoms and signs of the vocal cord dysfunction demonstrate very little variability with time. We suggest that as part of this disorder, a syndrome of chronic unremitting symptoms may occur.

(Thorax 1996;51:965-966)
\end{abstract}

Keywords: functional upper airways obstruction, vocal cord dysfunction, persistent symptoms.

The syndrome of functional upper airways obstruction (FAO) due to vocal cord dysfunction is now well described in the medical literature. First described by Patterson et al in 1974, it classically occurs paroxysmally and imitates acute asthmatic attacks, often in patients with co-existing mild asthma. ${ }^{23} \mathrm{We}$ present two cases which demonstrate a more chronic form of this disorder.

\section{Case history 1}

An 18 year old woman was admitted as a tertiary referral for investigation of chronic steroid dependent asthma. She had a history of mild childhood asthma which required little in the way of treatment until the age of 16 . Over the subsequent two and a half years she developed severe asthma-like symptoms with breathlessness, cough, and audible wheeze. Initially these symptoms were intermittent with acute exacerbations for which she was admitted and treated with high dose inhaled bronchodilators, oral and intravenous steroids, and aminophylline. All of these symptoms had become fixed over the preceding six months with only minor fluctuations in their intensity. She had spent over $90 \%$ of the preceding year as a hospital inpatient.

On transfer she was on regular treatment with oral prednisolone $40 \mathrm{mg}$ daily, intravenous aminophylline infusion, nebulised bronchodilators, and inhaled steroids. Clinical examination identified her to be cushingoid in appearance with fixed audible stridor and an accompanying dysphonia. Investigations showed her to be mildly atopic with normal lung volumes but she was unable to reproduce flow volume loops. Fibreoptic bronchoscopy using no sedation demonstrated fixed tight adduction of the true vocal cords with only a partial response to $10 \mathrm{mg}$ intravenous midazolam. A diagnosis of FAO due to paradoxical vocal cord motion was made, although the extent of the underlying asthma could not be assessed.

Over the subsequent four weeks all treatment was discontinued while she was monitored for evidence of asthma, with the exception of $7.5 \mathrm{mg}$ oral prednisolone because of adrenocortical suppression. Despite withdrawal of these medications her symptoms have remained the same and have shown little variability. Speech therapy has been commenced and she is subsequently making a slow but clear symptomatic improvement.

\section{Case history 2}

A 47 year old woman was referred urgently to the outpatient department by her general practitioner with a four month history of increasing shortness of breath and wheeze which had been treated as asthma. This had begun with a flu-like illness and had not responded to inhaled terbutaline, salmeterol, fluticasone (in a dose of $1 \mathrm{mg}$ twice daily through a large volume spacer) and a course of oral prednisolone. She was a smoker of 10 cigarettes per day. There was no history of childhood or previous adult respiratory problems, nor a family history of asthma and atopy. Her general practitioner had reported her to have constant "wheezing". However, physical examination was normal other than showing inspiratory stridor. The stridor was a little variable but was present with each breath, and the patient and her husband reported this had been present for the length of her illness.

Chest radiography, arterial blood gas tensions, and resting ECG were all normal. Spirometric tests were poorly performed but flow volume loops showed a reduction in the expiratory trace with a normal and consistent shape. The inspiratory loop, however, was flattened with a peak inspiratory flow rate of $12 \%$ predicted on repeated manoeuvres. Fibreoptic bronchoscopy showed tight adduction of the true vocal cords during inspiration only, with abduction during expiration. Vocal cord movement was normal during phonation. The inspiratory vocal cord adduction was abolished with a bolus of $5 \mathrm{mg}$ intravenous midazolam and this effect lasted for a period of four weeks following the procedure. During this time all inhalers were withdrawn with no worsening of her shortness of breath. Unfortunately, three days before reattending the outpatient department for follow up her symptoms recurred in their original form. Speech therapy is ongoing with only minor improvement to date.

\section{Discussion}

FAO is characterised by inappropriate vocal cord adduction which may occur during inspiration, expiration, or both. ${ }^{4}$ This may lead to audible respiratory sounds and sometimes a misdiagnosis of asthma. Whilst it is known to 
be a chronic syndrome in the sense that patients usually have recurrent "attacks", sometimes over a period of years, our cases demonstrate that it may also occur in a chronic form with relatively stable symptoms and signs.

Classically, FAO occurs in young women and seems to be commoner in health care workers. $^{256}$ There may be a past history of psychological disturbance ${ }^{28}$ and a past or current history suggestive of asthma. ${ }^{2}{ }^{3}$ It usually presents with acute attacks of breathlessness and audible stridor or wheeze, initiated by minor infections or emotional stress which may be resistant to conventional anti-asthma medications. If the diagnosis is not made, inappropriate treatment may be administered to the patient, including oral steroids and intravenous bronchodilators. In severe cases where hypoxaemia is present, artificial ventilation may be used with its associated physical and psychological morbidity.

Diagnosis is often difficult but a high index of clinical suspicion should be maintained in patients whose "asthma" is proving difficult to control. Peak flow levels are usually variable with dips often to unrecordable levels during an attack. Lung function testing between attacks is usually entirely normal. A firm diagnosis can be made at bronchoscopy when tight adduction of the vocal cords is demonstrated. Bronchoscopic findings may be missed if sedatives or atropine-like drugs (including nebulised anticholinergics) are given before bronchoscopy (since these have been shown to abolish the vocal cord adduction).${ }^{9}{ }^{10}$ Flow volume loops during attacks show evidence of extrathoracic airways obstruction during inspiration and/or expiration. ${ }^{4}$

There are a number of features typical of FAO in the cases we present. Both are female, and both had been misdiagnosed as suffering from asthma prior to referral. Both had been treated with steroids and bronchodilators with little effect, and case 1 was noted to be cushingoid on referral. Both patients had obvious inspiratory stridor, both had normal resting lung volumes, and case 2 had classical changes of extrathoracic airways obstruction on the inspiratory flow volume loop. Tight adduction of the vocal cords during inspiration was demonstrated bronchoscopically in both cases. A response to intravenous sedatives was also noted in both cases with partial response in case 1 and complete abolition of symptoms for a period of weeks in case 2 . In both cases withdrawal of anti-asthma medications was possible without a worsening of symptoms.

A number of atypical features are, however, also evident. Neither patient was in a paramedical occupation, neither had a history of psychological or psychiatric morbidity, and case 2 was older than one would expect for classical FAO. Perhaps the most interesting aspect of these two cases was the length of history of stable unremitting symptoms (four and 12 months, respectively). Detailed history from the patients and their relatives revealed that the symptoms experienced showed remarkably little day to day or week to week variation during the course of their illnesses.

1 Patterson R. Schatz M, Horton M. Munchhausen's stridor: non-organic laryngeal obstruction. Clin Allergy 1974; 4:307-10.

2 Nolan MT, Gibney N, Brennan N, Fitzgerald MX. Paradoxical vocal cord motion in asthma. Thorax 1985 ; 40:689.

3 Niven RM, Roberts T, Pickering CAC, Webb AK. Functional upper airway obstruction presenting as asthma. Respir Med 1992;86:513-6.

4 Goldman J, Muers M. Vocal cord dysfunction and wheezing. Thorax 1991;46:401-4.

5 Collett PW, Brancatisano T, Engel LA. Spasmodic croup in the adult. Am Rev Respir Dis 1983;127:500-4.

6 Downing ET, Braman SS, Fox MJ, Corroa WM. Factitious asthma. $\mathcal{F} A M A$ 1982;284:2878-81.

7 Brown TM, Merritt WD, Evans DL. Psychogenic vocal cord dysfunction masquerading as asthma. $\mathcal{F}$ Nerv Mental Dis 1988;176:308-10.

8 Rodenstein DO, Francis C, Stanescu DC. Emotional laryngeal wheezing: a new syndrome. Am Rev Respir Dis 1983; 127:354-6.

9 Appleblatt NH, Baker SR. Functional upper airway obstruction. A new syndrome. Arch Otolaryngol 1981; obstruction.

10 Cormier YF, Camus P, Desmeules MJ. Non-organic acute upper airway obstruction: description and a diagnostic approach. Am Rev Respir Dis 1980;121:147-50. 